\title{
THE FIRST COHOMOLOGY OF SEPARABLY RATIONALLY CONNECTED VARIETIES
}

\author{
FRANK GOUNELAS
}

We call a variety rationally connected if there passes a rational curve through every two general points and separably rationally connected if there exists a morphism $f: \mathbb{P}^{1} \rightarrow X$ such that $f^{*} T_{X}$ is an ample vector bundle. In characteristic zero these notions coincide, whereas they differ in characteristic $p$. Over an algebraically closed field of characteristic zero, a smooth projective separably rationally connected variety $X$ has $H^{i}\left(X, \mathcal{O}_{X}\right)=0$ for $i>0$ from Hodge theory (see [Deb03, p. 249]). In a recent preprint, Biswas and dos Santos [BdS11] prove a result which easily implies that in arbitrary characteristic, at least $H^{1}\left(X, \mathcal{O}_{X}\right)=0$.

Theorem. ([BdS11, Theorem 1.1]) Let $X$ be a smooth projective separably rationally connected variety over $k$ an algebraically closed field. Let $E$ be a vector bundle over $X$ such that for each $k$-morphism $f: \mathbb{P}^{1} \rightarrow X$, the pullback $f^{*} E$ is trivial. Then $E$ itself is trivial.

The claim on vanishing of first cohomology can be seen as follows. Pick a class in $H^{1}\left(X, \mathcal{O}_{X}\right)=\operatorname{Ext}^{1}\left(\mathcal{O}_{X}, \mathcal{O}_{X}\right)$ corresponding to a vector bundle $E$ of rank two. After pulling back to any $f: \mathbb{P}^{1} \rightarrow X$, we obtain

$$
0 \rightarrow \mathcal{O}_{\mathbb{P}^{1}} \rightarrow f^{*} E \rightarrow \mathcal{O}_{\mathbb{P}^{1}} \rightarrow 0 .
$$

It follows that $f^{*} E$ is split since $H^{1}\left(\mathbb{P}^{1}, \mathcal{O}_{\mathbb{P}^{1}}\right)=0$. Now from the main theorem in [BdS11], $E$ must itself be trivial. In positive characteristic we give another (cohomological) proof that at least $H^{1}\left(X, \mathcal{O}_{X}\right)=0$, which is a special case of the following.

Theorem. Let $X$ be a smooth projective variety over an algebraically closed field $k$ and $f: C \rightarrow X$ a morphism from a smooth projective curve such that $f^{*} T_{X}$ is an ample bundle. Then $H^{1}\left(X, \mathcal{O}_{X}\right)=0$.

It should be noted that not much is known about the groups $H^{i}\left(X, \mathcal{O}_{X}\right)$ for smooth separably rationally connected varieties where $i>1$ in positive characteristic. In the case of smooth Fano threefolds, Shepherd-Barron [SB97, Corollary 1.5] proved that $H^{i}\left(X, \mathcal{O}_{X}\right)=0$ for $i>0$. It is also shown (ibid. Corollary 12.4) that at least in the case of Picard rank one, Fano threefolds are liftable to characteristic zero so they are separably rationally connected (in general Fano varieties are only rationally chain connected) and hence satisfy the conditions of the theorem above. Smooth separably unirational (hence separably rationally connected) threefolds have been shown (see $\left[\mathrm{Nyg} 78\right.$, Theorem 2.5]) to have $H^{i}\left(X, \mathcal{O}_{X}\right)=0$ for $i=1,2,3$. In higher dimension, Kollár [Kol95, Theorem 11] has shown that there exist smooth Fano varieties in positive characteristic which are not even separably uniruled (see also [Kol96, Chapter V]), yet a general Fano hypersurface is so by [Zhu11, Theorem 1.4].

Date: April 22, 2014. 
On the other hand, Fano varieties which are also liftable to $W_{2}(k)$ satisfy Kodaira vanishing by Deligne-Illusie and hence have $H^{i}\left(X, \mathcal{O}_{X}\right)=0$ for $i>0$, but it is not known whether Fano varieties satisfy Kodaira vanishing (see [Kol13, Remark 3.5]).

For the proof of the main theorem, we proceed as follows. Similarly to the case of $C=\mathbb{P}^{1}$, one proves $H^{0}\left(X, \Omega_{X}^{m}\right)=0$ for $m>0$ (see [Gou12, Proposition 7.4]), essentially by noting that we can cover $X$ by the images of embeddings (see [Kol96, Theorem II.1.8]) from $C$ where the restriction $\left.T_{X}\right|_{C}$ is ample. Over $\mathbb{C}$ the theorem now follows as in the case of $\mathbb{P}^{1}$ from Hodge theory. Note that a theorem of Bogomolov and MacQuillan ([BM01], [KSCT07]) in characteristic zero proves that the existence of a curve satisfying the conditions of the theorem implies the existence of a very free $f: \mathbb{P}^{1} \rightarrow X$. In positive characteristic however this is not known, nor is it known that $X$ is rationally connected (see [Gou12] for a discussion in this direction). One can construct examples of $f: C \rightarrow X$ with $f^{*} T_{X}$ ample by starting with a very free curve $\mathbb{P}^{1} \rightarrow X$ and precomposing with a finite map $C \rightarrow \mathbb{P}^{1}$. In fact in dimension three and above, a general deformation of such a morphism $f$ will be an embedding (see [Kol96, Theorem II.1.8]).

The main structure of our proof in positive characteristic follows mutatis mutandis from the proof of Theorem 2.1 in Nygaard's paper [Nyg78]. First note that we may restrict to the algebraic closure of a finite field by spreading out over a suitable finitely generated $\mathbb{F}_{p}$-algebra $A$ and noting that $f: C \rightarrow X$ having $f^{*} T_{X}$ as an ample bundle is an open condition in the setting over $\operatorname{Spec} A$. Hence assume $f: C \rightarrow X$ with $f^{*} T_{X}$ ample is defined over $\overline{\mathbb{F}}_{p}$. Consider now the Artin-Schreier sequence of étale sheaves on $X$

$$
0 \rightarrow \mathbb{F}_{p} \rightarrow \mathbb{G}_{a} \stackrel{F-1}{\longrightarrow} \mathbb{G}_{a} \rightarrow 0 .
$$

The cohomologies of $\mathbb{G}_{a}$ and $\mathcal{O}_{X}$ agree and since the latter is coherent, étale and Zariski cohomology agree hence we may assume that all cohomology groups are taken in the étale site. We obtain an exact sequence

$$
0 \rightarrow H^{1}\left(X, \mathbb{F}_{p}\right) \rightarrow H^{1}\left(X, \mathcal{O}_{X}\right) \stackrel{F-1}{\longrightarrow} H^{1}\left(X, \mathcal{O}_{X}\right) \rightarrow 0
$$

where the last map is surjective due to SGA7.XXII Proposition 1.2. Suppressing base points, we use a method of Suwa [Suw83] to show that a $p$-group in the étale fundamental group $\pi_{1}(X)$ is trivial. In the case of $C=\mathbb{P}^{1}$ Kollár has proved that $\pi_{1}(X)$ is trivial using the de Jong-Starr Theorem, see [Deb03, Corollaire 3.6] (also [BdS11, Remark 2.5] for a correction), although in the case of higher genus $C$ the étale fundamental group could a priori be infinite (the author expects this is not the case however). Suwa, using a computation in crystalline cohomology, first proves that the vanishing of global differential forms implies that $h_{p}^{i}=\operatorname{dim} H^{i}\left(X, \mathbb{Q}_{p}\right)=0$ for $i>0$ from which $\chi_{p}(X)=\sum_{i}(-1)^{i} h_{p}^{i}=1$. The proof is identical in our setup. Note that pulling back $f: C \rightarrow X$ under an étale cover $Y \rightarrow X$ gives a smooth projective curve (possibly of higher genus) $g: C^{\prime} \rightarrow Y$ with $g^{*} T_{Y}$ also ample. Now, let $\pi_{1}(X) \rightarrow G$ be any finite quotient, $Y \rightarrow X$ the finite étale cover corresponding to $G$ and let $Y \rightarrow Z$ be the degree $p^{r}$ subcover corresponding to a $p$-Sylow in $G$. From the discussion before, both $Y$ and $Z$ admit morphisms from curves whose pullback of the tangent bundle is ample and so have $\chi_{p}=0$. By Crew's formula, $\chi_{p}(Y)=p^{r} \chi_{p}(Z)$ hence $p^{r}=\operatorname{deg}(Y / Z)=1$. Hence $\pi_{1}(X)$ has no elements of order 
$p$ (see [CL04] for a similar argument). Now, since $\pi_{1}(X)$ is profinite we obtain $\pi_{1}^{a b}(X) \otimes_{\mathbb{Z}} \mathbb{F}_{p}=0$.

Now $H^{1}\left(X, \mathbb{F}_{p}\right)=\operatorname{Hom}\left(\pi_{1}(X), \mathbb{F}_{p}\right)=\operatorname{Hom}\left(\pi_{1}^{a b}(X) \otimes \mathbb{F}_{p}, \mathbb{F}_{p}\right)=0$ and by SGA7.XXII Proposition 2.2.5, the semi-simple component of $H^{1}\left(X, \mathcal{O}_{X}\right)$ under the endomorphism induced by Frobenius $F$ is isomorphic to $H^{1}\left(X, \mathbb{F}_{p}\right) \otimes \overline{\mathbb{F}}_{p}$, which is trivial. Hence $F$ is nilpotent on $H^{1}\left(X, \mathcal{O}_{X}\right)$. The injectivity of the map of the corresponding sheaves induces $H^{0}\left(X, \mathcal{O}_{X} / F \mathcal{O}_{X}\right) \rightarrow H^{0}\left(X, \Omega_{X}^{1}\right)=0$ and so from the cohomology of the short exact sequence

$$
0 \rightarrow \mathcal{O}_{X} \stackrel{F}{\rightarrow} \mathcal{O}_{X} \rightarrow \mathcal{O}_{X} / F \mathcal{O}_{X} \rightarrow 0
$$

we obtain that $F: H^{1}\left(X, \mathcal{O}_{X}\right) \rightarrow H^{1}\left(X, \mathcal{O}_{X}\right)$ is injective. Since $F$ is thus injective and nilpotent on first cohomology, the result follows.

Acknowledgements. I would like to thank Jakob Stix for helpful conversations and to Hélène Esnault for some comments and bringing [BdS11] to my attention.

\section{REFERENCES}

[BdS11] I. Biswas and J. P. P. dos Santos, Triviality criteria for vector bundles over rationally connected varieties. Preprint, 2011.

[BM01] Feodor A. Bogomolov and Michael L. MacQuillan, Rational curves on foliated varieties. IHES, Preprint, 2001.

[CL04] Antoine Chambert-Loir. Points rationnels et groupes fondamentaux: applications de la cohomologie $p$-adique (d'après P. Berthelot, T. Ekedahl, H. Esnault, etc.). Astérisque, (294):viii, 125-146, 2004.

[Deb03] Olivier Debarre. Variétés rationnellement connexes (d'après T. Graber, J. Harris, J. Starr et A. J. de Jong). Astérisque, (290):Exp. No. 905, ix, 243-266, 2003. Séminaire Bourbaki. Vol. 2001/2002.

[Gou12] F. Gounelas, Free curves on varieties, preprint, arXiv:1208.4055 (2012).

[KSCT07] Stefan Kebekus, Luis Solá Conde, and Matei Toma. Rationally connected foliations after Bogomolov and McQuillan. J. Algebraic Geom., 16(1):65-81, 2007.

[Kol95] János Kollár. Nonrational hypersurfaces. J. Amer. Math. Soc., 8(1):241-249, 1995.

[Kol96] János Kollár. Rational curves on algebraic varieties, volume 32 of Ergebnisse der Mathematik und ihrer Grenzgebiete. 3. Folge. A Series of Modern Surveys in Mathematics [Results in Mathematics and Related Areas. 3rd Series. A Series of Modern Surveys in Mathematics]. Springer-Verlag, Berlin, 1996.

[Kol13] János Kollár. Singularities of the minimal model program, volume 200 of Cambridge Tracts in Mathematics. Cambridge University Press, Cambridge, 2013. With a collaboration of Sándor Kovács.

[Nyg78] Niels Nygaard. On the fundamental group of a unirational 3-fold. Invent. Math., 44(1):7586, 1978.

[SB97] N. I. Shepherd-Barron. Fano threefolds in positive characteristic. Compositio Math., 105(3):237-265, 1997.

[Suw83] Noriyuki Suwa. A note on the fundamental group of a unirational variety. Proc. Japan Acad. Ser. A Math. Sci., 59(3):98-99, 1983.

[Zhu11] Yi Zhu. Fano hypersurfaces in positive characteristic. Preprint, 2011.

Frank Gounelas, Institut für Mathematik, Humboldt Universität Zu Berlin, Unter den Linden 6, 10099 BerLin. 